\title{
Simplified tuning of long-range corrected density functionals for use in symmetry-adapted perturbation theory
}

\author{
Montgomery Gray and John M. Herbert* \\ Department of Chemistry and Biochemistry, The Ohio State University, Columbus, Ohio 43210 USA
}

(Dated: June 8, 2021)

\begin{abstract}
Long considered a failure, second-order symmetry-adapted perturbation theory (SAPT) based on Kohn-Sham orbitals, or SAPT(KS), can been resurrected for semiquantitative purposes using longrange corrected (LRC) density functionals whose asymptotic behavior is adjusted separately for each monomer. As in other contexts, correct asymptotic behavior can be enforced via "optimal tuning" of LRC functionals, based on the ionization energy theorem, but the tuning procedure is tedious, expensive for large systems, and comes with a troubling dependence on system size. Here, we show that essentially identical results are obtained using an automated tuning procedure based on the size of the exchange hole, making tuned "SAPT $(\omega \mathrm{KS})$ " fast and convenient. In conjunction with SAPT-based methods that sidestep second-order dispersion, this procedure achieves benchmarkquality interaction energies, along with the usual SAPT energy decomposition, without the hassle of system-specific tuning.
\end{abstract}

\section{INTRODUCTION}

Symmetry-adapted perturbation theory (SAPT) is the foremost $a b$ initio theory of intermolecular interactions. ${ }^{1-5}$ SAPT is a physically-meaningful energy decomposition analysis (EDA) for intermolecular interactions, yet one that is capable of benchmark-quality interaction energies if extended to sufficiently high order. The lowest-order variant, known as "SAPT0", ${ }^{2}$ uses HartreeFock wave functions for the monomers in conjunction with second-order perturbation theory to describe the intermolecular interactions. Combined with appropriate basis sets, SAPT0 is a semiquantitative treatment of intermolecular interactions with $\mathcal{O}\left(N^{5}\right)$ computational cost. $^{6}$ The description of strong hydrogen bonds can be improved through the use of Kohn-Sham orbitals from a density functional theory (DFT) calculation, albeit at the expense of (further) degrading the description of dispersion,,$^{7,8}$ which is already marginal at second order in perturbation theory. ${ }^{6}$ For this reason, the "SAPT(KS)" approach, meaning SAPT0 with KohnSham orbitals, was considered and rejected a long time ago. ${ }^{9-13}$ The method can be salvaged, however, through the use of exchange-correlation functionals with correct asymptotic behavior..$^{8,14}$

Perhaps more importantly, benchmark-quality interaction energies can be achieved using alternative combinations of DFT with SAPT that replace second-order dispersion with a more accurate formulation. The most widely used of these alternative formulations is DFTSAPT, ${ }^{4,15}$ also known as SAPT(DFT) ${ }^{3,16}$ which employs frequency-dependent density susceptibilities for the monomers (computed with DFT) to obtain the dispersion energy. In conjunction with density fitting techniques, DFT-SAPT is an $\mathcal{O}\left(N^{5}\right)$ method, ${ }^{15,17}$ albeit with a much larger prefactor as compared to SAPT0. Ab initio disper-

\footnotetext{
*herbert@chemistry.ohio-state.edu
}

sion potentials ${ }^{18-21}(\mathrm{SAPT}+a i \mathrm{D})$ or the many-body dispersion method ${ }^{22,23}$ (SAPT + MBD) also avoid secondorder dispersion and these methods achieve accuracies of $\lesssim 1 \mathrm{kcal} / \mathrm{mol}$ for benchmark noncovalent problems, ${ }^{20-22}$ with $\mathcal{O}\left(N^{3}\right)$ scaling. ${ }^{21}$ All of these DFT-based SAPT methods require the use of density functionals that are asymptotically correct. What that entails is described in Section II, and a convenient means to this end is the topic of the present work.

\section{THEORY}

The asymptotic behavior of an exact exchangecorrelation (xc) potential ought to be

$$
v_{\mathrm{xc}}(r) \sim v_{\mathrm{xc}}(\infty)-\frac{1}{r}
$$

for large $r$. The limiting value as $r \rightarrow \infty$ is

$$
v_{\mathrm{xc}}(\infty)=\mathrm{IE}+\varepsilon_{\mathrm{HOMO}}
$$

where IE $=E(N-1)-E(N)$ is the ionization energy and $\varepsilon_{\text {HOMO }}$ is the energy level of the highest-occupied molecular orbital (HOMO). ${ }^{24}$ In the context of DFT-SAPT, correct asymptotic behavior has generally been grafted onto a standard semilocal approximation for $v_{\mathrm{xc}},{ }^{24-26}$ whereas in SAPT(KS) the correct asymptotic behavior has usually been achieved using long-range corrected (LRC) density functionals. ${ }^{8}$ Correct asymptotic shape, i.e., the condition $v_{\mathrm{xc}}(r) \sim-1 / r$, is achieved automatically if the exchange functional is $100 \%$ Hartree-Fock exchange in the limit $r \rightarrow \infty$. We use the term LRC-DFT to indicate the subset of range-separated hybrid functionals that satisfy this asymptotic condition..$^{27-32}$

The correct asymptotic value of the potential is not guaranteed but can be set by adjusting the rangeseparation parameter $\omega$ such that $\operatorname{IE}(\omega)=-\varepsilon_{\text {HOMO }}(\omega)$, according to the ionization energy theorem in DFT. ${ }^{33,34}$ Especially in the context of time-dependent (TD-)DFT, 
this procedure has come to be called "optimal tuning", 32 and is widely used to correct the behavior of TD-DFT for charge-transfer excitations. ${ }^{32-35}$ The same tuning procedure has been used for SAPT(KS) calculations, ${ }^{8,14,18-21}$ and herein we will denote the range-separation parameter that satisfies the IE criterion as $\omega_{\mathrm{IE}}$ :

$$
\operatorname{IE}\left(\omega_{\mathrm{IE}}\right)=-\varepsilon_{\mathrm{HOMO}}\left(\omega_{\mathrm{IE}}\right) \text {. }
$$

Although widely used, this "optimal" or IE-tuning procedure has two significant problems, one practical and the other fundamental. Fundamentally, it is problematic in small-gap systems, as demonstrated by results presented herein. This is a more serious limitation than one might at first imagine, because semilocal functionals tend (anomalously) toward vanishing gaps for large systems. ${ }^{36-41}$ More pragmatically, the procedure is timeconsuming and therefore acts as a deterrent for potential users of SAPT(KS)-based methods.

In the present work, we explore the use of a black-box alternative to set $\omega$ based on the size of the exchange hole, in what has been called "global density-dependent" (GDD) tuning. ${ }^{42}$ Here, the range separation parameter is set to a value

$$
\omega_{\mathrm{GDD}}=C\left\langle d_{\mathrm{x}}^{2}\right\rangle^{-1 / 2}
$$

in which $d_{\mathrm{x}}^{2}$ is the second moment of the distance to the center of the exchange hole, ${ }^{43}$ and $\left\langle d_{\mathrm{x}}^{2}\right\rangle$ is a weighted average:

$$
\left\langle d_{\mathrm{x}}^{2}\right\rangle=\frac{\int \rho(\mathbf{r}) w(\mathbf{r}) d_{\mathrm{x}}^{2}(\mathbf{r}) d \mathbf{r}}{\int \rho(\mathbf{r}) w(\mathbf{r}) d \mathbf{r}} .
$$

The weighting function, ${ }^{42}$

$$
w(\mathbf{r})=\left\{\begin{array}{ll}
1, & t(\mathbf{r}) \leq \mu \\
0, & t(\mathbf{r})>\mu
\end{array},\right.
$$

is itself defined in terms of the function

$$
t(\mathbf{r})=\frac{\tau_{\mathrm{UEG}}(\mathbf{r})}{\tau(\mathbf{r})}
$$

where $\tau(\mathbf{r})$ is the kinetic energy density and $\tau_{\text {UEG }}(\mathbf{r})=$ $3\left(6 \pi^{2}\right)^{2 / 3} \rho(\mathbf{r})^{5 / 3} / 5$ is its value for the uniform electron gas. The orbital localization function $t(\mathbf{r})$ was originally introduced by Becke, ${ }^{44,45}$ who used it to construct both the electron localization function" ${ }^{44}$ and the "localized orbital locator". ${ }^{45}$ In the present context, $t(\mathbf{r})$ provides a weighting function such that $w(\mathbf{r}) \approx 0$ in the region of localized orbitals, so that $\omega_{\mathrm{GDD}}$ is set based on the asymptotics. ${ }^{42}$ The parameter $\mu$ in Eq. (6) is determined self-consistently such that the denominator in Eq. (5) equals unity. Finally, $C$ in Eq. (4) is an empirical parameter that is fit to reproduce the IE tuning condition in Eq. (3), for a set of small molecules. ${ }^{21,42,46}$

Both $w(\mathbf{r})$ and $d_{\mathrm{x}}^{2}(\mathbf{r})$ are functionals of $\rho(\mathbf{r})$. As described in Ref. 42 , the procedure is to first perform a selfconsistent LRC-DFT calculation, e.g., using LRC- $\omega \mathrm{PBE}$ with $\omega=0.3$ bohr $^{-1}$, which is an empirically-optimized "best guess" for the range separation parameter. ${ }^{28-30}$ That self-consistent density is then used to evaluate $\omega_{\text {GDD }}$ in Eq. (4), and these results are found to be negligibly different from a fully self-consistent procedure. ${ }^{42}$ In the context of SAPT(KS), the GDD-tuning method obviates the need to perform IE tuning separately for each monomer. Some isolated comparisons of IE- versus GDD-tuning for SAPT calculations were reported in Ref. 21, but here we report systematic comparisons for standard benchmark data sets of supramolecular complexes.

\section{COMPUTATIONAL METHODS}

We will use the term SAPT(KS) to refer exclusively to the second-order method that is generally called SAPT0 when used with Hartree-Fock (HF) monomers. ${ }^{2}$ For consistency, SAPT0 results are labeled as "SAPT $(\mathrm{HF})$ " in what follows, and will be compared alongside methods such as SAPT(B3LYP) and SAPT(LRC- $\omega$ PBE) that use different self-consistent field (SCF) methods to obtain the orbitals. We will also test "extended" SAPT (XSAPT) methods, ${ }^{20,21}$ in which the monomer wave functions are taken from charge-embedded "XPol" calculations ${ }^{7,47,48}$ and the second-order treatment of dispersion $\left(E_{\text {disp }}^{(2)}+E_{\text {exch-disp }}^{(2)}\right)$ is replaced by either atomatom dispersion potentials fitted to ab initio dispersion data (XSAPT $+a i \mathrm{D} 3),{ }^{20}$ or else a version of the manybody dispersion (MBD) model, ${ }^{49,50} \mathrm{XSAPT}+\mathrm{MBD} \cdot{ }^{22,23}$ XSAPT calculations reported here use CM5 embedding charges. $^{23}$

For the underlying LRC-DFT functional we mainly use LRC- $\omega \mathrm{PBE},{ }^{29}$ although some calculations with LRC$\mu \mathrm{BOP}^{28,31}$ are reported in the Supplementary Material. The parameter $C=0.885$ in Eq. (4) was determined in previous work, ${ }^{21}$ following Ref. 42 and using the same test set of small molecules. The best-fit value of $C$ varies significantly with the fraction of short-range Hartree-Fock exchange but is only weakly sensitive to basis set. ${ }^{46}$ Values $C \approx 0.9$ are appropriate when the self-consistent LRC- $\omega$ PBE calculation is performed using $\omega=0.3$ bohr $^{-1},{ }^{21,42}$ as is used here. All calculations were performed using Q-Chem. ${ }^{51}$ Values of $\omega_{\mathrm{IE}}$ and $\omega_{\mathrm{GDD}}$ for each monomer in each of the systems considered here can be found in the Supplementary Material.

\section{RESULTS \& DISCUSSION}

\section{A. IE tuning for small-gap systems}

We first demonstrate a looming problem with the IEbased tuning procedure for small-gap systems, using a homologous sequence of linear acenes: benzene, naphthalene, anthracene, tetracene, .... As the number of rings increases, the Kohn-Sham gap for these one-dimensional 


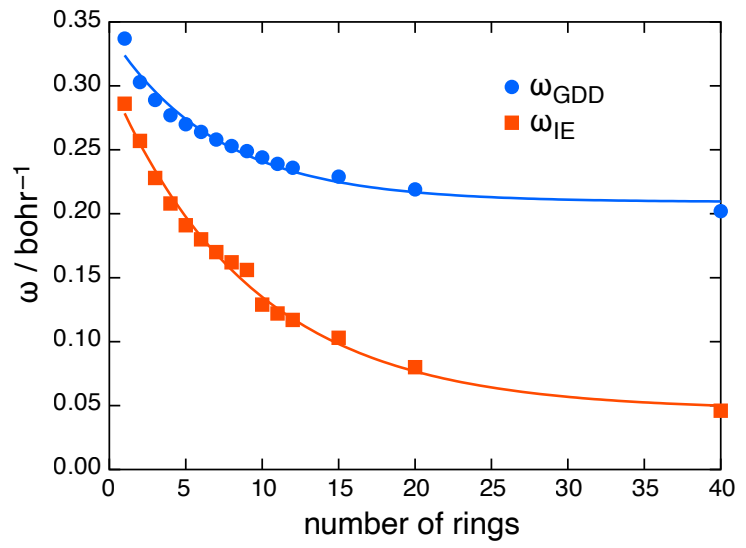

Fig. 1: Tuned values of $\omega$ for the linear acenes (benzene, naphthalene, anthracene, ...), computed at the LRC- $\omega \mathrm{PBE} /$ def2-TZVP level of theory. Results for LRC- $\mu \mathrm{BOP}$ are similar; see Figure S1.

acene nanoribbons decreases much faster as compared to that of their saturated (cyclohexane-based) analogues, the perhydroacenes. ${ }^{52}$

Figure 1 compares tuned values $\omega_{\mathrm{IE}}$ and $\omega_{\mathrm{GDD}}$ obtained for acenes with up to to 40 rings, demonstrating that both tuning procedures predict an optimal value of $\omega$ that decreases monotonically with system size. In the context of IE tuning, similar trends with increasing system size have been noted previously for conjugated $\pi$ systems ${ }^{42,53-55}$ for linear alkanes, ${ }^{42}$ for pentacene $/ \mathrm{C}_{60}$ clusters ${ }^{56}$ and for $\left(\mathrm{H}_{2} \mathrm{O}\right)_{n}^{-}$clusters. ${ }^{57}$ In the present data, we note that the asymptotic value of $\omega_{\mathrm{GDD}}$ is much larger than the asymptotic value of $\omega_{\text {IE }}$. For the 40-ring acene we obtain $\omega_{\mathrm{IE}}=0.046 \mathrm{bohr}^{-1}$, corresponding to a functional in which full HF exchange is activated on a length scale of $\sim 1 / \omega_{\text {IE }}=22$ bohr. While this is much shorter than the length of the 40-acene ribbon, it is much larger than the length scale of a chemical bond, so from the standpoint of the dynamical correlation that contributes to thermochemistry, the resulting LRC functional is operationally semilocal. In contrast, the GDD procedure converges to $\omega_{\mathrm{GDD}} \approx 0.20 \mathrm{bohr}^{-1}$ for the longest acene nanoribbons, which is within the range of statisticallyoptimized values of $\omega$ for LRC functionals. ${ }^{28-30}$

Note also what appears to be a small discontinuity in the progression of $\omega_{\mathrm{IE}}$ values between nonacene and decacene, such that the data for $n<10$ rings appear to extrapolate to a smaller asymptotic value of $\omega_{\mathrm{IE}}$ as compared to the data for $n \geq 10$. This discontinuity appears also (at the same system size) when LRC- $\mu \mathrm{BOP}$ is used instead of LRC- $\omega$ PBE; see Fig. S1 in the Supplementary Material. This small jump in $\omega_{\mathrm{IE}}$ may be related to the emergence of an open-shell singlet biradicaloid ground state of the linear acenes as the number of rings increases. ${ }^{58,59}$ In any case, in combination with the vanishing of the HOMO/LUMO gap for large systems described by semilocal functionals, ${ }^{36-41}$ these data present compelling evidence of an imminent problem with the IE tuning procedure as system size increases.

\section{B. Evaluation of SAPT(KS) methods}

As a baseline and starting point for further discussion, Table 1 reports error statistics for the S66 data set of small dimers, ${ }^{60}$ obtained using a variety of SAPT(KS) methods. Results are tabulated both with and without a " $\delta \mathrm{HF}$ " correction, ${ }^{2,20}$

$\delta \mathrm{HF}=E_{\mathrm{int}}^{\mathrm{HF}}-\left(E_{\text {elst }}^{(1)}+E_{\text {exch }}^{(1)}+E_{\text {ind,resp }}^{(2)}+E_{\text {exch-ind,resp }}^{(2)}\right)$.

This term consists of a counterpoise-corrected dimer $\mathrm{HF}$ calculation, to obtain $E_{\mathrm{int}}^{\mathrm{HF}}$, from which the nondispersion parts of the second-order SAPT interaction energy are subtracted. The result is an approximate correction for infinite-order induction. Note that the $\delta \mathrm{HF}$ correction should use HF theory even for SAPT(KS) methods based on DFT, because only in the HF case can this correction be strictly classified as induction, whereas a supramolecular DFT calculation would mix together different energy components in a manner that would be difficult to separate. Finally, all of the SAPT(KS) calculations in Table 1 employ the jun-cc-pVDZ basis set, a compromise choice that affords reasonable error cancellation in the second-order dispersion term. ${ }^{6}$

Error statistics in Table 1 are separated into three subsets of S66: hydrogen-bonded dimers, dispersiondominated dimers, and dimers where the interactions are mixed. This classification is based upon benchmark values of the electrostatic energy $\left(E_{\text {elst }}\right)$ and the dispersion energy $\left(E_{\text {disp }}\right) .{ }^{60,61}$ The hydrogen-bonded subset consists of dimers for which $\left|E_{\text {elst }}\right| \geq 2\left|E_{\text {disp }}\right|$, which includes dimers composed of water, methanol, methylamine, and acetic acid. The dispersion-dominated complexes are characterized by $\left|E_{\text {disp }}\right| \geq 2\left|E_{\text {elst }}\right|$, which includes dimers drawn from benzene, pyridine, ethene, ethyne, and some larger hydrocarbons. Other dimers (e.g., benzene-ethyne, ethyne-water, and benzene-acetic acid) are classified as "mixed", meaning that they do not satisfy either of the aforementioned criteria. There are 23 dimers categorized as hydrogen-bonded, 23 as dispersiondominated, and 20 classified as mixed. ${ }^{60}$

The SAPT $(\mathrm{HF})$ and SAPT $(\mathrm{HF})+\delta \mathrm{HF}$ results in Table 1 establish a baseline for what can be accomplished at low cost with traditional SAPT. We also consider SAPT(B3LYP), which was the lone representative of SAPT in a side-by-side comparison of different EDAs. ${ }^{62}$ Although SAPT(B3LYP) does offer a modest reduction in errors for hydrogen-bonded complexes, as compared to $\mathrm{SAPT}(\mathrm{HF})$, those gains are wiped out once the $\delta \mathrm{HF}$ correction is added to both methods. Furthermore, incorrect asymptotic behavior of B3LYP increases the errors for the dispersion-bound complexes, relative to the traditional SAPT $(\mathrm{HF})$ approach. Overall, the B3LYPbased approach is outperformed by $\mathrm{SAPT}(\mathrm{HF})+\delta \mathrm{HF}$. 
Table 1: Error statistics (in $\mathrm{kcal} / \mathrm{mol}$ ) for $\mathrm{SAPT}(\mathrm{KS})$ methods applied to the S66 database $^{60}$ and three subsets thereof.

\begin{tabular}{|c|c|c|c|c|c|c|c|c|}
\hline \multirow{2}{*}{ Method $^{a}$} & \multicolumn{2}{|c|}{ H-Bonded } & \multicolumn{2}{|c|}{ Disp.-Bound } & \multicolumn{2}{|c|}{ Mixed } & \multicolumn{2}{|c|}{ All S66 } \\
\hline & $\mathrm{MAE}^{b}$ & $\operatorname{Max}^{c}$ & $\mathrm{MAE}^{b}$ & $\operatorname{Max}^{c}$ & $\mathrm{MAE}^{b}$ & $\operatorname{Max}^{c}$ & $\mathrm{MAE}^{b}$ & $\operatorname{Max}^{c}$ \\
\hline $\mathrm{SAPT}(\mathrm{HF})$ & 2.20 & 6.14 & 0.95 & 1.93 & 0.78 & 1.77 & 1.33 & 6.14 \\
\hline $\mathrm{SAPT}(\mathrm{HF})+\delta \mathrm{HF}$ & 0.35 & 0.85 & 0.64 & 1.54 & 0.40 & 1.41 & 0.48 & 1.54 \\
\hline SAPT(B3LYP) & 1.49 & 4.24 & 1.49 & 3.93 & 0.66 & 1.36 & 1.24 & 4.24 \\
\hline $\mathrm{SAPT}(\mathrm{B} 3 \mathrm{LYP})+\delta \mathrm{HF}$ & 0.64 & 2.14 & 1.74 & 4.51 & 0.78 & 1.73 & 1.07 & 4.51 \\
\hline $\mathrm{SAPT}(\mathrm{LRC}-\omega \mathrm{PBE})^{d}$ & 2.87 & 7.69 & 0.91 & 2.04 & 0.71 & 3.60 & 1.53 & 7.69 \\
\hline $\mathrm{SAPT}(\mathrm{LRC}-\omega \mathrm{PBE})^{d}+\delta \mathrm{HF}$ & 0.97 & 2.27 & 0.95 & 2.04 & 0.50 & 2.66 & 0.82 & 2.66 \\
\hline $\mathrm{XSAPT}^{d}+a i \mathrm{D} 3$ & 2.01 & 5.76 & 0.15 & 0.54 & 0.25 & 0.64 & 0.83 & 5.76 \\
\hline $\mathrm{XSAPT}^{d}+a i \mathrm{D} 3+\delta \mathrm{HF}$ & 0.19 & 0.42 & 0.42 & 1.05 & 0.50 & 1.04 & 0.36 & 1.05 \\
\hline $\mathrm{XSAPT}^{d}+\mathrm{MBD}$ & 2.00 & 5.76 & 0.15 & 0.54 & 0.25 & 0.64 & 0.83 & 5.76 \\
\hline $\mathrm{XSAPT}^{d}+\mathrm{MBD}+\delta \mathrm{HF}$ & 0.18 & 0.75 & 0.46 & 1.11 & 0.53 & 1.07 & 0.39 & 1.11 \\
\hline
\end{tabular}

${ }^{a}$ Basis set is jun-cc-pVDZ for SAPT(KS) and def2-TZVPPD for XSAPT.

${ }^{b}$ Mean absolute error, with respect to complete-basis CCSD $(\mathrm{T})$ benchmarks. ${ }^{60}$

${ }^{c}$ Maximum deviation with respect to the benchmarks.

${ }^{d}$ Uses LRC- $\omega$ PBE with $\omega_{\text {GDD }}$ tuning.

Whereas the authors of Ref. 62 conclude that the "best" EDAs are those based on supramolecular DFT, this assessment was based on a skewed evaluation of SAPT methods that failed to consider $\mathrm{SAPT}(\mathrm{HF})+\delta \mathrm{HF}$, let alone any SAPT(KS) approach with proper asymptotic behavior. While "best" is highly subjective, the separation of energy components is better defined in SAPT(KS) as compared to supramolecular DFT. ${ }^{63-65}$

Larger errors for dispersion-dominated dimers that are observed with the asymptotically-incorrect SAPT(B3LYP) approach are mostly mitigated by using tuned SAPT(LRC- $\omega$ PBE), bringing them more in line with dispersion errors incurred by the traditional SAPT(HF) approach. As such, it is the second-order treatment of dispersion, rather than anything related to the density functional, that represents the primary source of error at the SAPT(LRC- $\omega \mathrm{PBE})+\delta \mathrm{HF}$ level. This confirms earlier results suggesting that $\omega$-tuning can put SAPT(KS) on par with SAPT(HF), ${ }^{8}$ so that the former is not nearly as problematic as early reports suggested, ${ }^{9-13}$ though neither does SAPT(KS) offer a significant advantage over the traditional HF-based approach. ${ }^{8}$ Both SAPT(KS) and SAPT(HF) require the $\delta \mathrm{HF}$ correction in order to achieve $\sim 1 \mathrm{kcal} / \mathrm{mol}$ accuracy for hydrogen bonds. Given that confusion persists over the proper functionals to use in SAPT(KS) ${ }^{62}$ we suggest that "SAPT $(\omega \mathrm{KS})$ " should be used to describe those methods that use monomer-specific $\omega$-tuning to enforce proper asymptotic behavior of $v_{\mathrm{xc}}$.

To move beyond second-order dispersion within proper SAPT requires methods with triple excitations and $\mathcal{O}\left(N^{7}\right)$ scaling. ${ }^{2,6}$ Alternatively, the perturbative treatment of dispersion can be replaced altogether, which is the unifying concept that underlies both DFT$\mathrm{SAPT}^{3,4}$ and XSAPT. ${ }^{20-22}$ Whereas DFT-SAPT is an $\mathcal{O}\left(N^{5}\right)$ method, ${ }^{15,17}$ XSAPT exhibits $\mathcal{O}\left(N^{3}\right)$ scaling. ${ }^{18,21}$
The performance of two variants, XSAPT $+a i \mathrm{D} 3$ and XSAPT + MBD, is characterized for the S66 data set in Table 1 . Without the $\delta \mathrm{HF}$ correct these methods exhibit $\sim 1 \mathrm{kcal} / \mathrm{mol}$ accuracy overall but the performance for the hydrogen-bonded subset is worse than that, with outliers approaching $6 \mathrm{kcal} / \mathrm{mol}$. With the $\delta \mathrm{HF}$ correction, the maximum error for the hydrogen-bonded complexes is reduced below $1 \mathrm{kcal} / \mathrm{mol}$. Both of these variants clearly outperform the other low-cost SAPT methods in Table 1 , although SAPT $(\mathrm{KS})+\delta \mathrm{HF}$, with the compromise jun-cc-pVDZ basis set, is surprisingly competitive. As will be seen below, that behavior is an artifact of the small size of the S66 dimers, which suppresses the dispersion term. ${ }^{21}$

\section{IE versus GDD tuning}

The main purpose of this work is to provide a side-byside comparison of the IE-based and GDD tuning procedures within the context of SAPT $(\omega \mathrm{KS})$-type methods. For S66, we select XSAPT $+\mathrm{MBD}+\delta \mathrm{HF}$ for this comparison because it affords the highest overall accuracy amongst the variants considered above, despite its reduced computational scaling. Table 2 compares error statistics for S66 using either IE or GDD tuning in several different basis sets. Previous work has demonstrated that triple- $\zeta$ basis sets are required to converge the electrostatic interactions, ${ }^{20}$ so it is not surprising to observe that the errors for hydrogen-bonded complexes decrease when double- $\zeta$ basis are replaced by triple- $\zeta$ ones, but it is pleasing to see that this also decreases both mean and maximum errors for the dispersion-dominated dimers. This situation should be contrasted with the use of juncc-pVDZ for SAPT0 calculations, for which electrostatic interactions are not fully converged. The choice of jun-cc- 
Table 2: Error statistics for S66 ${ }^{60}$ computed at the XSAPT $+\mathrm{MBD}+\delta \mathrm{HF}$ level using the tuned LRC- $\omega$ PBE functional.

\begin{tabular}{|c|c|c|c|c|c|c|c|c|c|}
\hline \multirow{3}{*}{ Tuning } & \multirow{3}{*}{ Basis Set } & \multicolumn{8}{|c|}{ Error (kcal/mol) } \\
\hline & & \multicolumn{2}{|c|}{ H-Bonded } & \multicolumn{2}{|c|}{ Disp.-Bound } & \multicolumn{2}{|c|}{ Mixed } & \multicolumn{2}{|c|}{ All S66 } \\
\hline & & $\mathrm{MAE}^{a}$ & $\operatorname{Max}^{6}$ & $\mathrm{MAE}^{a}$ & $\operatorname{Max}^{b}$ & $\mathrm{MAE}^{a}$ & $\operatorname{Max}^{b}$ & $\mathrm{MAE}^{a}$ & $\operatorname{Max}^{b}$ \\
\hline$\overline{\omega_{\mathrm{IE}}}$ & def2-SVPD & 1.13 & 3.21 & 1.62 & 2.98 & 1.33 & 2.36 & 1.37 & 3.21 \\
\hline$\omega_{\mathrm{GDD}}$ & def2-SVPD & 1.07 & 3.35 & 1.67 & 3.16 & 1.37 & 2.48 & 1.37 & 3.35 \\
\hline$\omega_{\mathrm{IE}}$ & def2-TZVP & 0.71 & 2.13 & 0.55 & 1.43 & 0.80 & 1.31 & 0.68 & 2.13 \\
\hline$\omega_{\mathrm{GDD}}$ & def2-TZVP & 0.65 & 1.20 & 0.55 & 1.60 & 0.85 & 1.41 & 0.68 & 1.60 \\
\hline$\omega_{\mathrm{IE}}$ & def2-TZVPP & 0.94 & 2.78 & 0.43 & 1.31 & 0.67 & 1.22 & 0.69 & 2.78 \\
\hline$\omega_{\mathrm{GDD}}$ & def2-TZVPP & 0.89 & 2.43 & 0.47 & 1.48 & 0.75 & 1.32 & 0.70 & 2.43 \\
\hline$\omega_{\mathrm{IE}}$ & def2 & 0.25 & 0.81 & 0.40 & 0.5 & 0.50 & 0.91 & 0.38 & 0.94 \\
\hline$\omega_{\mathrm{GDD}}$ & def2-TZVPD & 0.21 & 0.57 & 0.45 & 1.18 & 0.57 & 1.06 & 0.40 & 1.18 \\
\hline$\omega_{\mathrm{IE}}$ & def2-TZVPPD & 0.24 & 1.36 & 0.42 & 0.97 & 0.47 & 0.93 & 0.37 & 1.36 \\
\hline$\omega_{\mathrm{GDD}}$ & def2-TZVPPD & 0.18 & 0.75 & 0.46 & 1.11 & 0.53 & 1.07 & 0.39 & 1.11 \\
\hline
\end{tabular}

${ }^{a}$ Mean absolute error, with respect to complete-basis $\operatorname{CCSD}(\mathrm{T})$ benchmarks. ${ }^{60}$

${ }^{b}$ Maximum absolute deviation.

pVDZ in that case is a compromise in order to avoid large errors in second-order dispersion as the basis-set limit is approached, but such compromises are not required with XSAPT-based methods that avoid second-order dispersion. The data in Table 2 also highlight the benefit of diffuse functions. In our experience, users frequently decline to employ diffuse functions (presumably for reasons of cost), but the concomitant sacrifice in accuracy is undeniable.

The key observation in the present work is the fact that errors incurred by GDD tuning are nearly identical to the IE-tuned results. On the basis of the S66 data, there would seem to be no reason to perform the more tedious IE tuning procedure, which is also rather expensive for the larger systems that are considered below. As a counterpoint, the ansatz for $\omega_{\mathrm{GDD}}$ in Eq. (4) was fitted to reproduce $\omega_{\mathrm{IE}}$ for small molecules, and the monomers that comprise the $\mathrm{S} 66$ dimers are quite small, with the largest being pyridine $\left(\mathrm{C}_{5} \mathrm{H}_{5} \mathrm{~N}\right)$, uracil $\left(\mathrm{C}_{4} \mathrm{H}_{4} \mathrm{~N}_{2} \mathrm{O}_{2}\right)$, and pentane $\left(\mathrm{C}_{5} \mathrm{H}_{12}\right)$. We next consider some larger systems.

The L7 data set ${ }^{66}$ consists of dispersion-bound complexes ranging in size from (guanine) $)_{3}$ up to coronene dimer, $\left(\mathrm{C}_{24} \mathrm{H}_{12}\right)_{2}$, and also circumcoronene $\left(\mathrm{C}_{54} \mathrm{H}_{18}\right)$ partnered with either adenine or else a guanine-cytosine base pair. Because the induction energies are small for these systems, the $\delta \mathrm{HF}$ correction makes little difference $(\lesssim 0.5 \mathrm{kcal} / \mathrm{mol})$, and in the absence of this correction, no supramolecular calculations are required using XSAPT. Interaction energies at the XSAPT + MBD/ def2-TZVPPD level are reported in Table 3, where they are compared to the newest set of complete-basis $\operatorname{CCSD}(\mathrm{T})$ benchmarks for L7. ${ }^{67}$

The maximum discrepancy between the $\omega_{\mathrm{IE}^{-}}$and $\omega_{\mathrm{GDD}}$-based XSAPT + MBD results is $0.6 \mathrm{kcal} / \mathrm{mol}$, for the complex between circumcoronene and guaninecytosine, although the difference between the two XSAPT calculations amounts to a mere $2 \%$ of the benchmark interaction energy $\left(E_{\text {int }}=-28.63 \mathrm{kcal} / \mathrm{mol}\right)$. The
Table 3: XSAPT + MBD/def2-TZVPPD interaction energies for the L7 data set ${ }^{66}$ of large dispersion-bound dimers.

\begin{tabular}{|c|c|c|c|c|}
\hline System $^{a}$ & Tuning & $\frac{E_{\text {int }}(\mathrm{kcc}}{\mathrm{XSAPT}}$ & $\frac{\mathrm{l} / \mathrm{mol})}{\text { error }^{b}}$ & $\begin{array}{c}\Delta E_{\text {int }}(\omega)^{c} \\
(\%)\end{array}$ \\
\hline$(\text { cor })_{2}$ & $\begin{array}{l}\omega_{\mathrm{IE}} \\
\omega_{\mathrm{GDD}}\end{array}$ & $\begin{array}{l}-20.11 \\
-20.59\end{array}$ & $\begin{array}{l}0.82 \\
0.34\end{array}$ & 2.3 \\
\hline (circor) $\cdots($ Ade $)$ & $\begin{array}{l}\omega_{\mathrm{IE}} \\
\omega_{\mathrm{GDD}}\end{array}$ & $\begin{array}{l}-16.01 \\
-16.41\end{array}$ & $\begin{array}{l}0.90 \\
0.50\end{array}$ & 2.4 \\
\hline (circor) $\cdots \mathrm{GC}$ & $\begin{array}{l}\omega_{\mathrm{IE}} \\
\omega_{\mathrm{GDD}}\end{array}$ & $\begin{array}{l}-26.43 \\
-27.07\end{array}$ & $\begin{array}{l}2.20 \\
1.56\end{array}$ & 2.2 \\
\hline$(\text { octadecane })_{2}$ & $\begin{array}{l}\omega_{\mathrm{IE}} \\
\omega_{\mathrm{GDD}}\end{array}$ & $\begin{array}{l}-12.31 \\
-12.35\end{array}$ & $\begin{array}{l}1.31 \\
1.35\end{array}$ & 0.3 \\
\hline$(\mathrm{GC})_{2}$ & $\begin{array}{l}\omega_{\mathrm{IE}} \\
\omega_{\mathrm{GDD}}\end{array}$ & $\begin{array}{l}-13.61 \\
-13.95\end{array}$ & $\begin{array}{l}0.07 \\
0.41\end{array}$ & 2.5 \\
\hline$(\text { Gua })_{3}$ & $\begin{array}{l}\omega_{\mathrm{IE}} \\
\omega_{\mathrm{GDD}}\end{array}$ & $\begin{array}{l}-2.08 \\
-2.17\end{array}$ & $\begin{array}{l}0.00 \\
0.09\end{array}$ & 4.1 \\
\hline$(\mathrm{Phe})_{3}$ & $\begin{array}{l}\omega_{\mathrm{IE}} \\
\omega_{\mathrm{GDD}}\end{array}$ & $\begin{array}{l}-21.84 \\
-22.19\end{array}$ & $\begin{array}{l}3.62 \\
3.27 \\
\end{array}$ & 1.4 \\
\hline $\mathrm{MAE}^{d}$ & $\begin{array}{l}\omega_{\mathrm{IE}} \\
\omega_{\mathrm{GDD}}\end{array}$ & & $\begin{array}{l}1.28 \\
1.07 \\
\end{array}$ & 2.2 \\
\hline
\end{tabular}

${ }^{a}$ cor $=$ coronene, circum $=$ circumcoronene, Ade $=$ adenine, $\mathrm{GC}=$ guanine:cytosine base pair, Gua = guanine, Phe $=$ phenylalanine.

${ }^{b} E_{\text {int }}^{\mathrm{CCSD}(\mathrm{T})}-E_{\mathrm{int}}^{\mathrm{XSAPT}}$, using benchmarks from Ref. 67

${ }^{c}$ Difference between $\omega_{\mathrm{IE}}$ and $\omega_{\mathrm{GDD}}$ versions of $E_{\mathrm{int}}^{\mathrm{XSAPT}}$, expressed as a percentage of $E_{\text {int }}^{\mathrm{CCD}(\mathrm{T})}$.

${ }^{d}$ Mean absolute error.

final column of Table 3 lists the difference between $\omega_{\mathrm{IE}^{-}}$ and $\omega_{\mathrm{GDD}}$-based interaction energies as a percentage of the benchmark value, and these differences are each $\lesssim 2 \%$ except for the most weakly-bound complex, (guanine) ${ }_{3}$, for which the difference is $4 \%$ of the benchmark.

In terms of absolute accuracy, the maximum $\mathrm{XSAPT}+\mathrm{MBD}$ errors are $3.6 \mathrm{kcal} / \mathrm{mol} \quad\left(\omega_{\mathrm{IE}}\right)$ and 
Table 4: Interaction energies for ellipticine bound to doublestranded DNA, computed using XSAPT + MBD.

\begin{tabular}{|c|c|c|c|}
\hline \multirow[b]{2}{*}{ Tuning } & \multirow{2}{*}{ Basis } & \multicolumn{2}{|c|}{$E_{\text {int }}(\mathrm{kcal} / \mathrm{mol})$} \\
\hline & & XSAPT & error $^{a}$ \\
\hline$\omega_{\text {IE }}$ & def2-SVPD & -54.22 & 15.62 \\
\hline$\omega_{\mathrm{GDD}}$ & def2-SVPD & -54.07 & 15.47 \\
\hline$\omega_{\mathrm{IE}}$ & def2-TZVP & -43.14 & 4.54 \\
\hline$\omega_{\mathrm{GDD}}$ & def2-TZVP & -42.89 & 4.29 \\
\hline$\omega_{\mathrm{IE}}$ & def2-TZVPP & -42.91 & 4.31 \\
\hline$\omega_{\mathrm{GDD}}$ & def2-TZVPP & -42.62 & 4.02 \\
\hline$\omega_{\mathrm{IE}}$ & def2-TZVPD & -40.64 & 2.04 \\
\hline$\omega_{\mathrm{GDD}}$ & def2-TZVPD & -40.44 & 1.84 \\
\hline
\end{tabular}

${ }^{a}$ With respect to a complete-basis $\operatorname{CCSD}(\mathrm{T})$ benchmark, $E_{\text {int }}=-38.6 \pm 2.2 \mathrm{kcal} / \mathrm{mol} .{ }^{67}$

$3.3 \mathrm{kcal} / \mathrm{mol}\left(\omega_{\mathrm{GDD}}\right)$, both for the phenylalanine trimer, while the mean absolute errors (MAEs) are $1.3 \mathrm{kcal} / \mathrm{mol}$ $\left(\omega_{\mathrm{IE}}\right)$ and $1.1 \mathrm{kcal} / \mathrm{mol}\left(\omega_{\mathrm{GDD}}\right)$. To put these numbers in perspective, the MAE for SAPT0/jun-cc-pVDZ as applied to the $\mathrm{L} 7$ data set is $4.8 \mathrm{kcal} / \mathrm{mol}$ and the maximum error is $10.3 \mathrm{kcal} / \mathrm{mol}^{21}$ As compared to the $\mathrm{S} 66$ results, this represents a stark divergence in the performance of SAPT0 relative to XSAPT, and it occurs due to the much larger dispersion energies for the L7 complexes, which reveal the failure of second-order dispersion, especially for $\pi-\pi$ interactions. ${ }^{20,21}$

As a final example, we consider a DNA intercalation complex with the anti-cancer drug ellipticine, which has become a standard benchmark problem for non-covalent interactions. ${ }^{21-23,67,68}$ Interaction energies computed at the XSAPT + MBD level are presented in Table 4, using several different basis sets and comparing $\omega_{\mathrm{IE}}$ and $\omega_{\mathrm{GDD}}$ versions in each case. The two tuning schemes never deviate from one another by more than $0.3 \mathrm{kcal} / \mathrm{mol}$. In terms of accuracy, the errors are reduced as the quality of the basis set is improved, and these calculations once again highlight the important role of diffuse functions: the XSAPT $+\mathrm{MBD} /$ def2-TZVPD interaction energies lie within the estimated uncertainties in the completebasis $\operatorname{CCSD}(\mathrm{T})$ benchmark, $\pm 2.2 \mathrm{kcal} / \mathrm{mol} .{ }^{67}$ For comparison, the best-available supramolecular DFT results for this system are $E_{\text {int }}=-41.3 \mathrm{kcal} / \mathrm{mol}(\mathrm{B} 97 \mathrm{M}-\mathrm{V} /$ def2-TZVPPD) and $E_{\text {int }}=-43.7 \mathrm{kcal} / \mathrm{mol}(\omega \mathrm{B} 97 \mathrm{M}-\mathrm{V} /$ def2-TZVPPD) ${ }^{23}$ corresponding to errors of 2.7 and $5.1 \mathrm{kcal} / \mathrm{mol}$, respectively. XSAPT $+\mathrm{MBD}$ is therefore more accurate but also cheaper, ${ }^{22}$ as it does not require a supersystem calculation.

\section{CONCLUSIONS}

In the context of SAPT(KS) and related XSAPT methods, we find that the GDD tuning scheme works equally well as compared to "optimal" IE-based tuning, but sidesteps the series of monomer calculations that are required for IE tuning. The GDD approach also avoids the size-dependent tuning catastrophe that afflicts IE tuning for small-gap systems, making it more robust in addition to being more convenient. Differences in interaction energies, when using one tuning scheme versus the other, are small in comparison to the absolute errors in interaction energies. Given its ease of use, GDD tuning should replace IE tuning for (X)SAPT calculations based on Kohn-Sham DFT, and indeed our group has mostly relied on the GDD scheme in recent work. ${ }^{21-23,69}$

This work also highlights (and reiterates ${ }^{8}$ ) the fact that SAPT(KS) methods should be based on asymptoticallycorrect functionals for best results. Methods such as SAPT(B3LYP), held up as an exemplar of a SAPT-based $\mathrm{EDA},{ }^{62}$ in fact misrepresent the accuracy of low-cost SAPT approaches. Correct asymptotic behavior is easily (and automatically) enforced using LRC functionals in conjunction with the tuning schemes examined herein. We suggest the name "SAPT $(\omega \mathrm{KS})$ " to refer to the subset of SAPT(KS) methods in which tuned LRC functionals are used to achieve proper behavior of $v_{\mathrm{xc}}$ for each monomer. In conjunction with alternatives to secondorder dispersion such as XSAPT $+\mathrm{MBD},{ }^{22,23}$ this affords a cubic-scaling method with $\sim 1 \mathrm{kcal} / \mathrm{mol}$ accuracy for non-covalent interaction energies, in systems large and small.

\section{Acknowledgments}

This work was supported by the U.S. Department of Energy, Office of Basic Energy Sciences, Division of Chemical Sciences, Geosciences, and Biosciences under Award No. DE-SC0008550. Calculations were performed at the Ohio Supercomputer Center under project no. PAA-0003. ${ }^{70}$ J.M.H. serves on the board of directors of Q-Chem Inc.

\section{Data availability statement}

The data that support the findings of this study are available from the corresponding author upon reasonable request. 
${ }^{1}$ K. Szalewicz, K. Patkowski, and B. Jeziorski, "Intermolecular interactions via perturbation theory: From diatoms to biomolecules", in Intermolecular Forces and Clusters II, D. J. Wales, Ed., Vol. 116 of Structure and Bonding; Springer-Verlag: Berlin, 2005; pages 43-117.

2 E. G. Hohenstein and C. D. Sherrill, "Wavefunction methods for noncovalent interactions", WIREs Comput. Mol. Sci. 2, 304-326 (2012).

3 K. Szalewicz, "Symmetry-adapted perturbation theory of intermolecular forces", WIREs Comput. Mol. Sci. 2, 254272 (2012).

4 G. Jansen, "Symmetry-adapted perturbation theory based on density functional theory for noncovalent interactions", WIREs Comput. Mol. Sci. 4, 127-144 (2014).

${ }^{5}$ K. Patkowski, "Recent developments in symmetry-adapted perturbation theory", WIREs Comput. Mol. Sci.. 10, e1452 (2020).

6 T. M. Parker, L. A. Burns, R. M. Parrish, A. G. Ryno, and C. D. Sherrill, "Levels of symmetry adapted perturbation theory (SAPT). I. Efficiency and performance for interaction energies", J. Chem. Phys. 140, 094106 (2014).

7 J. M. Herbert, L. D. Jacobson, K. U. Lao, and M. A. Rohrdanz, "Rapid computation of intermolecular interactions in molecular and ionic clusters: Self-consistent polarization plus symmetry-adapted perturbation theory", Phys. Chem. Chem. Phys. 14, 7679-7699 (2012).

${ }^{8}$ K. U. Lao and J. M. Herbert, "Symmetry-adapted perturbation theory with Kohn-Sham orbitals using nonempirically tuned, long-range-corrected density functionals", J. Chem. Phys. 140, 044108 (2014).

9 H. L. Williams and C. F. Chabalowski, "Using Kohn-Sham orbitals in symmetry-adapted perturbation theory to investigate intermolecular interactions", J. Phys. Chem. A 105, 646-659 (2001).

10 G. Jansen and A. Heßelmann, "Comment on "Using KohnSham orbitals in symmetry-adapted perturbation theory to investigate intermolecular interactions" ", J. Phys. Chem. A 105, 11156-11157 (2001).

11 A. J. Misquitta and K. Szalewicz, "Intermolecular forces from asymptotically corrected density functional description of monomers", Chem. Phys. Lett. 357, 301-306 (2002).

12 A. Heßelmann and G. Jansen, "First-order intermolecular interaction energies from Kohn-Sham orbitals", Chem. Phys. Lett. 357, 464-470 (2002).

13 A. Heßelmann and G. Jansen, "Intermolecular induction and exchange-induction energies from coupled-perturbed Kohn-Sham density functional theory", Chem. Phys. Lett. 362, 319-325 (2002).

14 M. Hapka, L. Rajchel, M. Modrzejewski, G. Chałasiǹski, and M. M. Szczȩśniak, "Tuned range-separated hybrid functionals in the symmetry-adapted perturbation theory", J. Chem. Phys. 141, 134120 (2014).

15 A. Heßelmann, G. Jansen, and M. Schütz, "Densityfunctional theory symmetry-adapted intermolecular perturbation theory with density fitting: A new efficient method to study intermolecular interaction energies", J. Chem. Phys. 122, 014103 (2005).

16 A. J. Misquitta and K. Szalewicz, "Symmetry-adapted perturbation-theory calculations of intermolecular forces employing density-functional description of monomers",
J. Chem. Phys. 122, 214109 (2005).

17 R. Bukowski, R. Podeszwa, and K. Szalewicz, "Efficient calculation of coupled Kohn-Sham dynamic susceptibility functions and dispersion energies with density fitting", Chem. Phys. Lett. 414, 111-116 (2005).

${ }^{18}$ K. U. Lao and J. M. Herbert, "Accurate intermolecular interactions at dramatically reduced cost: XPol+SAPT with empirical dispersion", J. Phys. Chem. Lett. 3, 3241-3248 (2012).

19 K. U. Lao and J. M. Herbert, "An improved treatment of empirical dispersion and a many-body energy decomposition scheme for the explicit polarization plus symmetryadapted perturbation theory (XSAPT) method", J. Chem. Phys. 139, 034107 (2013). Erratum: J. Chem. Phys. 140, 119901 (2014).

${ }^{20}$ K. U. Lao and J. M. Herbert, "Accurate and efficient quantum chemistry calculations of noncovalent interactions in many-body systems: The XSAPT family of methods", J. Phys. Chem. A 119, 235-253 (2015).

21 K. U. Lao and J. M. Herbert, "Atomic orbital implementation of extended symmetry-adapted perturbation theory (XSAPT) and benchmark calculations for large supramolecular complexes", J. Chem. Theory Comput. 14, 2955-2978 (2018)

22 K. Carter-Fenk, K. U. Lao, K.-Y. Liu, and J. M. Herbert, "Accurate and efficient ab initio calculations for supramolecular complexes: Symmetry-adapted perturbation theory with many-body dispersion", J. Phys. Chem. Lett. 10, 2706-2714 (2019).

${ }^{23}$ K.-Y. Liu, K. Carter-Fenk, and J. M. Herbert, "Selfconsistent charge embedding at very low cost, with application to symmetry-adapted perturbation theory", J. Chem. Phys. 151, 031102 (2019).

24 D. J. Tozer and N. C. Handy, "Improving virtual KohnSham orbitals and eigenvalues: Application to excitation energies and static polarizabilities", J. Chem. Phys. 109, 10180-10189 (1998).

${ }^{25}$ R. van Leeuwen and E. J. Baerends, "Exchange-correlation potential with correct asymptotic behavior", Phys. Rev. A 49, 2421-2431 (1994).

26 M. Grüning, O. V. Gritsenko, S. J. A. van Gisbergen, and E. J. Baerends, "Shape corrections to exchange-correlation potentials by gradient-regulated seamless connection of model potentials for inner and outer region", J. Chem. Phys. 114, 652-660 (2001).

27 A. W. Lange, M. A. Rohrdanz, and J. M. Herbert, "Charge-transfer excited states in a $\pi$-stacked adenine dimer, as predicted using long-range-corrected timedependent density functional theory", J. Phys. Chem. B 112, 6304-6308 (2008). Erratum: J. Phys. Chem. B 112, 7345 (2008).

28 M. A. Rohrdanz and J. M. Herbert, "Simultaneous benchmarking of ground- and excited-state properties with longrange-corrected density functional theory", J. Chem. Phys. 129, 034107 (2008).

29 M. A. Rohrdanz, K. M. Martins, and J. M. Herbert, "A long-range-corrected density functional that performs well for both ground-state properties and time-dependent density functional theory excitation energies, including chargetransfer excited states", J. Chem. Phys. 130, 054112 (2009). 
${ }^{30}$ A. W. Lange and J. M. Herbert, "Both intra- and interstrand charge-transfer excited states in B-DNA are present at energies comparable to, or just above, the ${ }^{1} \pi \pi^{*}$ excitonic bright states", J. Am. Chem. Soc. 131, 3913-3922 (2009).

31 R. M. Richard and J. M. Herbert, "Time-dependent density-functional description of the ${ }^{1} \mathrm{~L}_{a}$ state in polycyclic aromatic hydrocarbons: Charge-transfer character in disguise?", J. Chem. Theory Comput. 7, 1296-1306 (2011).

32 B. Alam, A. F. Morrison, and J. M. Herbert, "Charge separation and charge transfer in the low-lying excited states of pentacene", J. Phys. Chem. C 124, 24653-24666 (2020).

33 T. Stein, L. Kronik, and R. Baer, "Reliable prediction of charge transfer excitations in molecular complexes using time-dependent density functional theory", J. Am. Chem. Soc. 131, 2818-2820 (2009).

${ }^{34}$ R. Baer, E. Livshits, and U. Salzner, "Tuned rangeseparated hybrids in density functional theory", Annu. Rev. Phys. Chem. 61, 85-109 (2010).

35 S. Kümmel, "Charge-transfer excitations: A challenge for time-dependent density functional theory that has been met", Adv. Energy Mater. 7, 1700440 (2017).

36 J. Antony and S. Grimme, "Fully ab initio protein-ligand interaction energies with dispersion corrected density functional theory", J. Comput. Chem. 33, 1730-1739 (2012).

37 E. Rudberg, "Difficulties in applying pure Kohn-Sham density functional theory electronic structure methods to protein molecules", J. Phys.: Condens. Matt. 24, 072202 (2012).

38 H. J. Kulik, N. Luehr, I. S. Ufimtsev, and T. J. Martinez, "Ab initio quantum chemistry for protein structures", J. Phys. Chem. B 116, 12501-12509 (2012).

39 C. M. Isborn, B. D. Mar, B. F. E. Curchod, I. Tavernelli, and T. J. Martínez, "The charge transfer problem in density functional theory calculations of aqueously solvated molecules", J. Phys. Chem. B 117, 12189-12201 (2013).

40 G. Lever, J. D. Cole, N. D. M. Hine, P. D. Haynes, and M. C. Payne, "Electrostatic considerations affecting the calculated HOMO-LUMO gap in protein molecules", J. Phys.: Condens. Matt. 25, 152101 (2013).

${ }^{41}$ D. J. Cole and N. D. M. Hine, "Applications of large-scale density functional theory in biology", J. Phys.: Condens. Matt. 28, 393001 (2016).

42 M. Modrzejewski, Ł. Rajchel, G. Chalasinski, and M. M. Szczesniak, "Density-dependent onset of the long-range exchange: A key to donor-acceptor properties", J. Phys. Chem. A 117, 11580-11586 (2013).

43 A. D. Becke and E. R. Johnson, "Exchange-hole dipole moment and the dispersion interaction", J. Chem. Phys. 122, 154104 (2005).

44 A. D. Becke and K. E. Edgecombe, "A simple measure of electron localization in atomic and molecular systems", J. Chem. Phys. 92, 5397-5403 (1990).

45 H. L. Schmider and A. D. Becke, "Chemical content of the kinetic energy density", J. Mol. Struct. (Theochem) 527, 51-61 (2000).

${ }^{46}$ K. Carter-Fenk, C. J. Mundy, and J. M. Herbert, "Natural charge-transfer analysis: Eliminating spurious chargetransfer states in time-dependent density functional theory via diabatization, with application to projection-based embedding" (DOI: 10.26434/chemrxiv.14485368.v1).

47 L. D. Jacobson and J. M. Herbert, "An efficient, fragmentbased electronic structure method for molecular systems: Self-consistent polarization with perturbative two-body exchange and dispersion", J. Chem. Phys. 134, 094118
(2011).

48 J. Gao, D. G. Truhlar, Y. Wang, M. J. M. Mazack, P. Löffler, M. R. Provorse, and P. Rehak, "Explicit polarization: A quantum mechanical framework for developing next generation force fields", Acc. Chem. Res. 47, 28372845 (2014).

49 A. Tkatchenko, R. A. DiStasio Jr., R. Car, and M. Scheffler, "Accurate and efficient method for many-body van der Waals interactions", Phys. Rev. Lett. 108, 236402 (2012).

50 A. Ambrosetti, A. M. Reilly, R. A. DiStasio Jr., and A. Tkatchenko, "Long-range correlation energy calculated from coupled atomic response functions", J. Chem. Phys. 140, 18A508 (2014).

51 Y. Shao, Z. Gan, E. Epifanovsky, A. T. B. Gilbert, M. Wormit, J. Kussmann, A. W. Lange, A. Behn, J. Deng, X. Feng, D. Ghosh, M. Goldey, P. R. Horn, L. D. Jacobson, I. Kaliman, R. Z. Khaliullin, T. Kús, A. Landau, J. Liu, E. I. Proynov, Y. M. Rhee, R. M. Richard, M. A. Rohrdanz, R. P. Steele, E. J. Sundstrom, H. L. Woodcock III, P. M. Zimmerman, D. Zuev, B. Albrecht, E. Alguire, B. Austin, G. J. O. Beran, Y. A. Bernard, E. Berquist, K. Brandhorst, K. B. Bravaya, S. T. Brown, D. Casanova, C.-M. Chang, Y. Chen, S. H. Chien, K. D. Closser, D. L. Crittenden, M. Diedenhofen, R. A. DiStasio Jr., H. Do, A. D. Dutoi, R. G. Edgar, S. Fatehi, L. Fusti-Molnar, A. Ghysels, A. Golubeva-Zadorozhnaya, J. Gomes, M. W. D. Hanson-Heine, P. H. P. Harbach, A. W. Hauser, E. G. Hohenstein, Z. C. Holden, T.-C. Jagau, H. Ji, B. Kaduk, K. Khistyaev, J. Kim, J. Kim, R. A. King, P. Klunzinger, D. Kosenkov, T. Kowalczyk, C. M. Krauter, K. U. Lao, A. Laurent, K. V. Lawler, S. V. Levchenko, C. Y. Lin, F. Liu, E. Livshits, R. C. Lochan, A. Luenser, P. Manohar, S. F. Manzer, S.-P. Mao, N. Mardirossian, A. V. Marenich, S. A. Maurer, N. J. Mayhall, C. M. Oana, R. Olivares-Amaya, D. P. O'Neill, J. A. Parkhill, T. M. Perrine, R. Peverati, P. A. Pieniazek, A. Prociuk, D. R. Rehn, E. Rosta, N. J. Russ, N. Sergueev, S. M. Sharada, S. Sharma, D. W. Small, A. Sodt, T. Stein, D. Stück, Y.-C. Su, A. J. W. Thom, T. Tsuchimochi, L. Vogt, O. Vydrov, T. Wang, M. A. Watson, J. Wenzel, A. White, C. F. Williams, V. Vanovschi, S. Yeganeh, S. R. Yost, Z.-Q. You, I. Y. Zhang, X. Zhang, Y. Zhao, B. R. Brooks, G. K. L. Chan, D. M. Chipman, C. J. Cramer, W. A. Goddard III, M. S. Gordon, W. J. Hehre, A. Klamt, H. F. Schaefer III, M. W. Schmidt, C. D. Sherrill, D. G. Truhlar, A. Warshel, X. Xu, A. Aspuru-Guzik, R. Baer, A. T. Bell, N. A. Besley, J.-D. Chai, A. Dreuw, B. D. Dunietz, T. R. Furlani, S. R. Gwaltney, C.-P. Hsu, Y. Jung, J. Kong, D. S. Lambrecht, W. Liang, C. Ochsenfeld, V. A. Rassolov, L. V. Slipchenko, J. E. Subotnik, T. Van Voorhis, J. M. Herbert, A. I. Krylov, P. M. W. Gill, and M. HeadGordon, "Advances in molecular quantum chemistry contained in the Q-Chem 4 program package", Mol. Phys. 113, 184-215 (2015).

${ }^{52}$ K. Carter-Fenk and J. M. Herbert, "Reinterpreting $\pi$ stacking", Phys. Chem. Chem. Phys. 22, 24870-24886 (2020)

53 T. Körzdörfer, J. S. Sears, C. Sutton, and J.-L. Brédas, "Long-range corrected hybrid functionals for $\pi$-conjugated systems: Dependence of the range-separation parameter on conjugation length", J. Chem. Phys. 135, 204107 (2011).

54 T. B. de Queiroz and S. Kümmel, "Charge-transfer excitations in low-gap systems under the influence of solvation 
and conformational disorder: Exploring range-separation tuning", J. Chem. Phys. 141, 084303 (2014).

55 J. Bois and T. Körzdörfer, "Size-dependence of nonempirically tuned DFT starting points for $G_{0} W_{0}$ applied to $\pi$ conjugated molecular chains", J. Chem. Theory Comput. 13, 4962-4971 (2017).

56 B. Yang, Y. Yi, C.-R. Zhang, S. G. Aziz, V. Coropceanu, and J.-L. Brédas, "Impact of electron delocalization on the nature of the charge-transfer states in model pentacene/C 60 interfaces: A density functional theory study", J. Phys. Chem. C 118, 27648-27656 (2014).

57 F. Uhlig, J. M. Herbert, M. P. Coons, and P. Jungwirth, "Optical spectroscopy of the bulk and interfacial hydrated electron from ab initio calculations", J. Phys. Chem. A 118, 7507-7515 (2014).

${ }^{58}$ M. Bendikov, H. M. Duong, K. Starkey, K. N. Houk, E. A. Carter, and F. Wudl, "Oligoacenes: Theoretical prediction of open-shell singlet diradical ground states", J. Am. Chem. Soc. 126, 7416-7417 (2004). Erratum: J. Am. Chem. Soc. 126, 10493 (2004).

${ }^{59}$ Y. Yang, E. R. Davidson, and W. Yang, "Nature of ground and electronic excited states of higher acenes", Proc. Natl. Acad. Sci. USA 113, E5098-E5107 (2016).

60 J. Řezác, K. E. Riley, and P. Hobza, "S66: A well-balanced database of benchmark interaction energies relevant to biomolecular structures", J. Chem. Theory Comput. 7, 2427-2438 (2011). Erratum: J. Chem. Theory Comput. 10, 1359-1360 (2014).

61 J. C. Flick, D. Kosenkov, E. G. Hohenstein, C. D. Sherrill, and L. V. Slipchenko, "Accurate prediction of noncovalent interaction energies with the effective fragment potential method: Comparison of energy components to symmetryadapted perturbation theory for the S22 test set", J. Chem. Theory Comput. 8, 2835-2843 (2012). Erratum: J. Chem. Theory Comput. 10, 4759-4760 (2014).

${ }^{62}$ M. J. S. Phipps, T. Fox, C. S. Tautermann, and C.-K. Skylaris, "Energy decomposition analysis approaches and their evaluation on prototypical protein-drug interaction patterns", Chem. Soc. Rev. 44, 3177-3211 (2015).

63 K. U. Lao and J. M. Herbert, "Energy decomposition analysis with a stable charge-transfer term for interpreting intermolecular interactions", J. Chem. Theory Comput. 12, 2569-2582 (2016).

${ }^{64}$ M. Shahbaz and K. Szalewicz, "Do semilocal densityfunctional approximations recover dispersion energies at small intermonomer separations?", Phys. Rev. Lett. 121, 113402 (2018).

65 J. Andrés, P. W. Ayers, R. A. Boto, R. Carbó-Dorca, H. Chermette, J. Cioslowski, J. Contreras-García, D. L. Cooper, G. Frenking, C. Gatti, F. Heidar-Zadeh, L. Joubert, A. M. Pendás, E. Matito, I. Mayer, A. J. Misquitta, Y. Mo, J. Pilmé, P. L. A. Popelier, M. Rahm, E. RamosCordoba, P. Salvador, W. H. E. Schwarz, S. Shahbazian, B. Silvi, M. Solà, K. Szalewicz, V. Tognetti, F. Weinhold, and E.-L. Zins, "Nine questions on energy decomposition analysis", J. Comput. Chem. 40, 2248-2283 (2019).

${ }^{66}$ R. Sedlak, T. Janowski, M. Pitoňák, J. Řezáč, P. Pulay, and P. Hobza, "Accuracy of quantum chemical methods for large noncovalent complexes", J. Chem. Theory Comput. 9, 3364-3374 (2013).

67 F. Ballesteros, S. Dunivan, and K. U. Lao, "Coupled cluster benchmarks of large noncovalent complexes: The L7 dataset as well as DNA-ellipticine and buckycatcherfullerene", J. Chem. Phys. 154, 154104 (2021).

68 A. Benali, L. Shulenburger, N. A. Romero, J. Kim, and O. A. von Lilienfeld, "Application of diffusion Monte Carlo to materials dominated by van der Waals interactions", J. Chem. Theory Comput. 10, 3417-3422 (2014).

69 K. U. Lao and J. M. Herbert, "A simple correction for nonadditive dispersion within extended symmetry-adapted perturbation theory (XSAPT)", J. Chem. Theory Comput. 14, 5128-5142 (2018).

70 Ohio Supercomputer Center, http://osc.edu/ark:/19495/f5s1ph73. 